\title{
Bi-slant $\xi^{\perp}$-Riemannian submersions
}

\author{
Sezin Aykurt Sepet (D) \\ Department of Mathematics, Arts and Science Faculty, Kırşehir Ahi Evran University, Kırşehir, Turkey
}

\begin{abstract}
We introduce bi-slant $\xi^{\perp}$-Riemannian submersions from Sasakian manifolds onto Riemannian manifolds as a generalization of slant and semi-slant $\xi^{\perp}$-Riemannian submersion and present some examples. We give the necessary and sufficient conditions for the integration of the distributions used to define the bi-slant $\xi^{\perp}$-Riemannian submersions and examine the geometry of foliations. After we obtain necessary and sufficient conditions related to totally geodesicness of such submersion. Finally we give some decomposition theorems for total manifold.
\end{abstract}

Mathematics Subject Classification (2020). 53C25, 53C43

Keywords. Riemannian submersion, Sasakian manifold, bi-slant $\xi^{\perp}$ - Riemannian submersion

\section{Introduction}

The differential geometry of slant submanifolds has been studied by many authors since B.Y Chen [10] defined slant immersions in complex geometry as a natural generalization of both holomorphic immersions and totally real immersions. Carriazo [9] has introduced bi-slant immersions. Then Uddin et al. [31] have studied warped product bi-slant immersions in Kaehler manifolds. As a generalization of CR-submanifolds, slant and semi-slant submanifolds, Cabrerizo et al. [8] have defined bi-slant submanifolds of almost contact metric manifolds. Recently, Alqahtani et al. [5] have investigated warped product bi-slant submanifolds of cosymplectic manifolds.

On the other hand Riemannian submersions were introduced by B. O'Neill [19] and A. Gray [12]. Since then Riemannian submersions have been studied extensively by many geometers. In [32], B. Watson defined almost Hermitian submersions between almost Hermitian manifolds. In this study, he investigated some geometric properties between base manifold and total manifold as well as fibers.

B. Sahin [27] described the notion slant submersion from almost Hermitian manifold onto an arbitrary Riemannian manifold as follows: Let $F$ be a Riemannian submersion from an almost Hermitian manifold $(M, g, J)$ onto a Riemannian manifold $\left(N, g^{\prime}\right)$. If for any nonzero vector $X \in \Gamma\left(\operatorname{ker} F_{*}\right)$ the angle $\theta(X)$ between $J X$ and $\Gamma\left(\operatorname{ker} F_{*}\right)$ is a constant, i.e. it does not dependent on the choice of $p \in M$ and $X \in \Gamma\left(\operatorname{ker} F_{*}\right)$, then it is called that $F$ is a slant submersion. Therefore the angle $\theta$ is said to be the slant angle of the slant submersion. Many interesting studies on several types of submersions have been

Email address: saykurt@ahievran.edu.tr

Received: 18.02.2021; Accepted: 31.07.2021 
done. For instance, slant and semi-slant submersions [3,13-16, 21], bi-slant submersions [23], quasi bi-slant submersions [22], anti-invariant Riemannian submersions [25, 28], semiinvariant submersions $[20,26]$, pointwise slant submersions $[6,18]$, hemi-slant submersions [30], Lagrangian submersions [29], generic submersions [24].

Furthermore J.W. Lee [17] defined anti-invariant $\xi^{\perp}$-Riemannian submersions from almost contact metric manifolds. Later Akyol et al studied the geometry of semi-invariant $\xi^{\perp}$-Riemannian submersion, semi-slant $\xi^{\perp}$-Riemannian submersions and conformal antiinvariant $\xi^{\perp}$-submersions from almost contact metric manifolds $[1,2,4]$.

The paper is regulated as following. In Section 2, we recall the basic formulas and concepts needed for this paper. In Section 3 we define bi-slant $\xi^{\perp}$-Riemannian submersions from Sasakian manifolds onto Riemannian manifolds and give some examples. We also examine the geometry of leaves of distributions and find necessary and sufficient conditions for such maps to be totally geodesic. In the last section, we obtain some decomposition theorems.

\section{Preliminaries}

An almost contact structure $(\phi, \xi, \eta)$ on a manifold $M$ of dimension $2 n+1$ is defined by a tensor field $\phi$ of type $(1,1)$, a vector field $\xi$ (Reeb vector field) and a 1-form $\eta$ so that

$$
\phi^{2}=-I+\eta \otimes \xi, \eta(\xi)=1, \eta \circ \phi=0, \phi \xi=0 .
$$

Here $I$ is the identity map of $T M$. There always exist a Riemannian metric $g$ on $M$ proving the following compatibility condition with the structure $(\phi, \xi, \eta)$

$$
g(\phi X, \phi Y)=g(X, Y)-\eta(X) \eta(Y)
$$

where $X, Y$ are arbitrary vector fields on $M$. Then the manifold $M$ with the structure $(\phi, \xi, \eta, g)$ is called an almost contact metric manifold. An almost contact metric manifold is named normal if

$$
[\phi, \phi]+2 d \eta \otimes \xi=0
$$

where $[\phi, \phi]$ is Nijenhuis tensor of $\phi$. Let $\Phi$ denote the 2-form on an almost contact metric manifold $(M, \phi, \xi, \eta, g)$ expressed with $\Phi(X, Y)=g(X, \phi Y)$ for any $X, Y \in \Gamma(T M)$. The $\Phi$ is called the fundamental 2 -form of $M$. An almost contact metric manifold $(M, \phi, \xi, \eta, g)$ is said to be a contact metric manifold if $\Phi=d \eta$. A normal contact metric manifold is called a Sasakian manifold. Then the structure equations of Sasakian manifold are given by

$$
\left(\nabla_{X} \phi\right) Y=g(X, Y) \xi-\eta(Y) X \text { and } \nabla_{X} \xi=-\phi X,
$$

where $\nabla$ is the Levi-Civita connection of $g$ and $X, Y \in \Gamma(T M)$.

Let $(M, g)$ and $\left(N, g^{\prime}\right)$ be a Riemannian manifolds with $m$ and $n$ dimension, respectively, such that $m>n$. A surjective mapping $F: M \longrightarrow N$ is said to be a Riemannian submersion if $F$ has maximal rank and the differential map $F_{*}$ restricted to $\Gamma\left(\left(\operatorname{ker} F_{*}\right)^{\perp}\right)$ is a linear isometry.

For any $q \in N, F^{-1}(q)$ which is an $m-n$ dimensional submanifold of $M$, called fiber. A vector field on $M$ is named vertical (or horizontal) if it is always tangent (or orthogonal) to the fibers[19]. A vector field $X$ on $M$ is named basic if $X \in \Gamma\left(\left(\operatorname{ker} F_{*}\right)^{\perp}\right)$ and $F_{*} X_{p}=X_{* F(p)}$ for all $p \in M[11]$.

A Riemannian submersion $F: M \longrightarrow N$ is qualified by two fundamental tensor fields $\mathcal{T}$ and $\mathcal{A}$ on $M$ such that

$$
\begin{aligned}
\mathcal{T}(E, F) & =\mathcal{T}_{E} F=\mathcal{H} \nabla_{\mathcal{V} E} \mathcal{V} F+\mathcal{V} \nabla_{\mathcal{V} E} \mathcal{H} F \\
\mathcal{A}(E, F) & =\mathcal{A}_{E} F=\mathcal{V} \nabla_{\mathcal{H} E} \mathcal{H} F+\mathcal{H} \nabla_{\mathcal{H} E} \mathcal{V} F
\end{aligned}
$$


where $E$ and $F$ are arbitrary vector fields on $M$ and $\nabla$ the Levi-Civita connection of $M$. In addition, for $X, Y \in \Gamma\left(\left(\operatorname{ker} F_{*}\right)^{\perp}\right)$ and $U, W \in \Gamma\left(\operatorname{ker} F_{*}\right)$ the tensor fields satisfy

$$
\begin{aligned}
& \mathcal{T}_{U} W=\mathcal{T}_{W} U \\
& \mathcal{A}_{X} Y=-\mathcal{A}_{Y} X=\frac{1}{2} \mathcal{V}[X, Y] .
\end{aligned}
$$

Moreover, note that a Riemannian submersion $F: M \longrightarrow N$ has totally geodesic fibers if and only if $\mathcal{T}$ vanishes identically. Now, let's remember the following lemma from [19].

Lemma 2.1. Let $F: M \longrightarrow N$ be a Riemannian submersion between Riemannian manifolds $(M, g)$ and $\left(N, g^{\prime}\right)$. For the basic vector fields $X, Y \in \Gamma(T M)$ we have

i) $g(X, Y)=g^{\prime}\left(X_{*}, Y_{*}\right) \circ F$,

ii) $F_{*}\left([X, Y]^{\mathcal{H}}\right)=\left[X_{*}, Y_{*}\right]$

iii) $[V, X]$ is vertical for $V \in \Gamma\left(\operatorname{ker} F_{*}\right)$,

iv) $\left(\nabla_{X}^{M} Y\right)^{\mathcal{H}}$ is a basic vector field corresponding to $\nabla_{X_{*}}^{N} Y_{*}$, where $\nabla^{M}$ and $\nabla^{N}$ are the Levi-Civita connections on $M$ and $N$, respectively.

Furhermore considering (2.4) and (2.5) we write

$$
\begin{aligned}
\nabla_{U} V & =\mathcal{T}_{U} V+\bar{\nabla}_{U} V \\
\nabla_{U} X & =\mathcal{H} \nabla_{U} X+\mathcal{T}_{U} X \\
\nabla_{X} U & =\mathcal{A}_{X} U+\mathcal{V} \nabla_{X} U \\
\nabla_{X} Y & =\mathcal{H}_{X} Y+\mathcal{A}_{X} Y
\end{aligned}
$$

where $X, Y \in \Gamma\left(\left(\operatorname{ker} F_{*}\right)^{\perp}\right), U, V \in \Gamma\left(\operatorname{ker} F_{*}\right)$ and $\bar{\nabla}_{U} V=\mathcal{V} \nabla_{U} V$.

Let $\psi: M \longrightarrow N$ is a smooth map. In that case the second fundamental form of $\psi$ is defined by

$$
\nabla \psi_{*}(X, Y)=\nabla_{X}^{\psi} \psi_{*}(Y)-\psi_{*}\left(\nabla_{X}^{M} Y\right)
$$

where $X, Y \in \Gamma(T M)$ and $\nabla^{\psi}$ the pullback connection. Note that $\psi$ is called harmonic if trace $\nabla \psi_{*}=0$ and $\psi$ is named as a totally geodesic map if $\left(\nabla \psi_{*}\right)(X, Y)=0$ for $X, Y \in \Gamma(T M)[7]$.

\section{Bi-slant $\xi^{\perp}$-Riemannian submersions}

Definition 3.1. Let $F$ is a Riemannian submersion from a Sasakian manifold $(M, \phi, \xi, \eta, g)$ onto a Riemannian manifold $\left(N, g^{\prime}\right)$ so that $\xi \in \Gamma\left(\left(\operatorname{ker} F_{*}\right)^{\perp}\right)$. Then $F: M \longrightarrow N$ is called a bi-slant $\xi^{\perp}$-Riemannian submersion if there exist a pair of the orthogonal distributions $D_{1} \subset$ ker $F_{*}$ and $D_{2} \subset \operatorname{ker} F_{*}$ such that

(1) $\operatorname{ker} F_{*}=D_{1} \oplus D_{2}$

(2) $D_{1}$ and $D_{2}$ are two slant distributions with the slant angles $\theta_{1}$ and $\theta_{2}$, respectively $F$ is called proper if its slant angles satisfy $\theta_{1}, \theta_{2} \neq 0, \frac{\pi}{2}$.

Note that $\mathbb{R}^{2 n+1}$ denote a Sasakian manifold with the structure $(\phi, \xi, \eta, g)$ defined as

$$
\begin{gathered}
\phi\left(\sum_{i=1}^{n}\left(X_{i} \frac{\partial}{\partial x^{i}}+Y_{i} \frac{\partial}{\partial y^{i}}\right)+Z \frac{\partial}{\partial z}\right)=\sum_{i=1}^{n}\left(Y_{i} \frac{\partial}{\partial x^{i}}-X_{i} \frac{\partial}{\partial y^{i}}\right), \\
\eta=\frac{1}{2}\left(d z-\sum_{i=1}^{n} y^{i} d x^{i}\right), \xi=2 \frac{\partial}{\partial z} \\
g=\eta \otimes \eta+\frac{1}{4} \sum_{i=1}^{n}\left(d x^{i} \otimes d x^{i}+d y^{i} \otimes d y^{i}\right),
\end{gathered}
$$


where $\left(x^{1}, \ldots, x^{n}, y^{1}, \ldots, y^{n}, z\right)$ are the Cartesian coordinates.

Now, considering the above definition, we can give the following example.

Example 3.2. Let $F: \mathbb{R}^{9} \longrightarrow \mathbb{R}^{5}$ be a submersion defined by

$$
F\left(x_{1}, x_{2}, x_{3}, x_{4}, y_{1}, y_{2}, y_{3}, y_{4}, z\right)=\left((\cos \alpha) x_{1}-(\sin \alpha) x_{2}, \frac{x_{3}+x_{4}}{\sqrt{2}},(\sin \beta) y_{1}+(\cos \beta) y_{2}, y_{3}, z\right)
$$

then

$$
\begin{array}{r}
\operatorname{ker} F_{*}=\operatorname{span}\left\{V_{1}=\sin \alpha \frac{\partial}{\partial x_{1}}+\cos \alpha \frac{\partial}{\partial x_{2}}, V_{2}=\frac{1}{\sqrt{2}}\left(\frac{\partial}{\partial x_{3}}-\frac{\partial}{\partial x_{4}}\right),\right. \\
\left.V_{3}=\cos \beta \frac{\partial}{\partial y_{1}}-\sin \beta \frac{\partial}{\partial y_{2}}, V_{4}=\frac{\partial}{\partial y_{4}}\right\}
\end{array}
$$

and

$$
\begin{array}{r}
\left(\operatorname{ker} F_{*}\right)^{\perp}=\operatorname{span}\left\{H_{1}=\cos \alpha \frac{\partial}{\partial x_{1}}-\sin \alpha \frac{\partial}{\partial x_{2}}, H_{2}=\frac{1}{\sqrt{2}}\left(\frac{\partial}{\partial x_{3}}+\frac{\partial}{\partial x_{4}}\right)\right. \\
\left.H_{3}=\sin \beta \frac{\partial}{\partial y_{1}}+\cos \beta \frac{\partial}{\partial y_{2}}, H_{4}=\frac{\partial}{\partial y_{3}}, \xi=\frac{\partial}{\partial z}\right\}
\end{array}
$$

Thus we obtain $D_{1}=\operatorname{span}\left\{V_{1}, V_{3}\right\}$ and $D_{2}=\operatorname{span}\left\{V_{2}, V_{4}\right\}$ with the angle $\cos \theta_{1}=$ $\sin (\beta-\alpha)$ and $\theta_{2}=\frac{\pi}{4}$. Then $F$ is a bi-slant $\xi^{\perp-R i e m a n n i a n ~ s u b m e r s i o n . ~}$

Example 3.3. Given a submersion $F: \mathbb{R}^{9} \longrightarrow \mathbb{R}^{5}$ by

$$
F\left(x_{1}, x_{2}, x_{3}, x_{4}, y_{1}, y_{2}, y_{3}, y_{4}, z\right)=\left(\frac{x_{1}+\sqrt{3} x_{4}}{2}, \sin \alpha x_{2}+\cos \alpha x_{3}, y_{1}, y_{3}, z\right)
$$

Then the submersion $\mathrm{F}$ is a bi-slant $\xi^{\perp}$-Riemannian submersion such that $D_{1}=\operatorname{span}\left\{V_{1}=\right.$ $\left.\frac{1}{2}\left(\sqrt{3} \frac{\partial}{\partial x_{1}}-\frac{\partial}{\partial x_{4}}\right), V_{4}=\frac{\partial}{\partial y_{4}}\right\}$ and $D_{2}=\operatorname{span}\left\{V_{2}=\cos \alpha \frac{\partial}{\partial x_{2}}-\sin \alpha \frac{\partial}{\partial x_{3}}, V_{3}=\frac{\partial}{\partial y_{2}}\right\}$ with slant angles $\theta_{1}=\frac{\pi}{3}$ and $\theta_{2}=\alpha$, respectively.

Let $F$ be a bi-slant $\xi^{\perp}$-Riemannian submersion from a Sasakian manifold $(M, \phi, \xi, \eta, g)$ onto a Riemannian manifold $\left(N, g^{\prime}\right)$. Then for $U \in \Gamma\left(\operatorname{ker} F_{*}\right)$, we have

$$
U=P U+Q U
$$

where $P U \in \Gamma\left(D_{1}\right)$ and $Q U \in \Gamma\left(D_{2}\right)$.

In addition, for $U \in \Gamma\left(\operatorname{ker} F_{*}\right)$, we get

$$
\phi U=\psi U+\omega U
$$

where $\psi U \in \Gamma\left(\operatorname{ker} F_{*}\right)$ and $\omega U \in \Gamma\left(\operatorname{ker} F_{*}\right)^{\perp}$.

Similarly, for $X \in \Gamma\left(\operatorname{ker} F_{*}\right)^{\perp}$, we can write

$$
\phi X=B X+C X
$$

where $B X \in \Gamma\left(\operatorname{ker} F_{*}\right)$ and $C X \in \Gamma\left(\operatorname{ker} F_{*}\right)^{\perp}$.

The horizontal distribution $\left(\operatorname{ker} F_{*}\right)^{\perp}$ is decompesed as

$$
\left(\operatorname{ker} F_{*}\right)^{\perp}=\omega D_{1} \oplus \omega D_{2} \oplus \mu
$$

where $\mu$ is the complementary distribution to $\omega D_{1} \oplus \omega D_{2}$ in $\left(\text { ker } F_{*}\right)^{\perp}$ and contains $\xi$. Also it is invariant distribution of $\left(\text { ker } F_{*}\right)^{\perp}$ with respect to $\phi$.

From (3.1), (3.2) and (3.3) we have following equations

$$
\psi D_{1}=D_{1}, \quad \psi D_{2}=D_{2}, \quad B \omega D_{1}=D_{1}, \quad B \omega D_{2}=D_{2} .
$$

Furthermore, by using the equations (2.1), (3.2) and (3.3) we get: 
Lemma 3.4. Let $F$ be a bi-slant $\xi^{\perp}$-Riemannian submersion from a Sasakian manifold $(M, \phi, \xi, \eta, g)$ onto a Riemannian manifold $\left(N, g^{\prime}\right)$. then we obtain
i) $\psi^{2} U+B \omega U=-U$
ii) $\omega \psi U+C \omega U=0$
iii) $\psi^{2} W+B \omega W=-W$
iv) $\omega \psi W+C \omega W=0$
v) $\omega B X+C^{2} X=-X+\eta(X) \xi$
vi) $\psi B X+B C X=0$
for any $U \in \Gamma\left(D_{1}\right), W \in \Gamma\left(D_{2}\right)$ and $X \in \Gamma\left(\left(\operatorname{ker} F_{*}\right)^{\perp}\right)$

On the other hand from (2.8), (2.9), (3.2) and (3.3) we have

$$
\begin{aligned}
& \left(\nabla_{U} \psi\right) V=B \mathcal{T}_{U} V-\mathcal{T}_{U} \omega V \\
& \left(\nabla_{U} \omega\right) V=C \mathcal{T}_{U} V-\mathcal{T}_{U} \psi V \\
& \left(\nabla_{U} \psi\right) V=\bar{\nabla}_{U} \psi V-\psi \bar{\nabla}_{U} V \\
& \left(\nabla_{U} \omega\right) V=\mathcal{H} \nabla_{U} \omega V-\omega \bar{\nabla}_{U} V
\end{aligned}
$$

for any $U, V \in \Gamma\left(\operatorname{ker} F_{*}\right)$. We say that $\omega$ is parallel if

$$
\left(\nabla_{U} \omega\right) V=0
$$

for $U, V \in \Gamma\left(\operatorname{ker} F_{*}\right)$.

Now we can give the following theorem by using Definition 3.1 and the equation (3.2).

Theorem 3.5. Let $F$ be a Riemannian submersion from a Sasakian manifold $(M, \phi, \xi, \eta, g)$ onto a Riemannian manifold $\left(N, g^{\prime}\right)$. Then $F$ is a bi-slant $\xi^{\perp}$-Riemannian submersion if and only if there exist slant angle $\theta_{i}$ defined on $D_{i}$ such that

$$
\psi^{2}=-\left(\cos ^{2} \theta_{i}\right) I, i=1,2
$$

Proof. The proof of this theorem is the similar to semi-slant submanifolds [8].

Theorem 3.6. Let $F$ be a bi-slant $\xi^{\perp}$-Riemannian submersion from a Sasakian manifold $(M, \phi, \xi, \eta, g)$ onto a Riemannian manifold $\left(N, g^{\prime}\right)$ with slant angles $\theta_{1}, \theta_{2}$. Then

i) $D_{1}$ is integrable if and only if

$$
\begin{aligned}
g\left(\mathcal{T}_{U} \omega \psi V-\mathcal{T}_{V} \omega \psi U, W\right)= & g\left(\mathcal{T}_{U} \omega V-\mathcal{T}_{V} \omega U, \psi W\right) \\
& +g\left(\mathcal{H} \nabla_{U} \omega V-\mathcal{H} \nabla_{V} \omega U, \omega W\right)
\end{aligned}
$$

ii) $D_{2}$ is integrable if and only if

$$
\begin{aligned}
g\left(\mathcal{T}_{W} \omega \psi Z-\mathcal{T}_{Z} \omega \psi W, U\right)= & g\left(\mathcal{T}_{W} \omega Z-\mathcal{T}_{Z} \omega W, \psi U\right) \\
& +g\left(\mathcal{H} \nabla_{W} \omega Z-\mathcal{H} \nabla_{Z} \omega W, \omega U\right)
\end{aligned}
$$

for $U, V \in \Gamma\left(D_{1}\right)$ and $W, Z \in \Gamma\left(D_{2}\right)$.

Proof. For $U, V \in \Gamma\left(D_{1}\right)$ and $X \in \Gamma\left(\left(\operatorname{ker} F_{*}\right)^{\perp}\right)$, since $g([U, V], X)=0$, it is sufficient to show $g([U, V], W)=0$ for $W \in \Gamma\left(D_{2}\right)$. Then since $M$ is a Sasakian manifold we get

$$
\begin{aligned}
g([U, V], W)= & -g\left(\nabla_{U} \phi \psi V, W\right)+g\left(\nabla_{U} \omega V, \phi W\right) \\
& +g\left(\nabla_{V} \phi \psi U, W\right)-g\left(\nabla_{V} \omega U, \phi W\right) .
\end{aligned}
$$

Theorem 3.5 and the equation (2.9) imply that

$$
\begin{aligned}
\sin ^{2} \theta_{1} g([U, V], W)= & -g\left(\mathcal{T}_{U} \omega \psi V-\mathcal{T}_{V} \omega \psi U, W\right)+g\left(\mathcal{T}_{U} \omega V-\mathcal{T}_{V} \omega U, \psi W\right) \\
& +g\left(\mathcal{H} \nabla_{U} \omega V-\mathcal{H} \nabla_{V} \omega U, \omega W\right)
\end{aligned}
$$

Similarly for $W, Z \in \Gamma\left(D_{2}\right)$ and $U \in \Gamma\left(D_{1}\right)$ it can be shown that

$$
\begin{aligned}
\sin ^{2} \theta_{2} g([W, Z], U)= & -g\left(\mathcal{T}_{W} \omega \psi Z-\mathcal{T}_{Z} \omega \psi W, U\right)+g\left(\mathcal{T}_{W} \omega Z-\mathcal{T}_{Z} \omega W, \psi U\right) \\
& +g\left(\mathcal{H}_{W} \omega Z-\mathcal{H}_{W} \nabla_{Z} \omega W, \omega U\right) .
\end{aligned}
$$


which proves $(i i)$. Thus the proof is completed.

Theorem 3.7. Let $F$ be a bi-slant $\xi^{\perp}$-Riemannian submersion from a Sasakian manifold $(M, \phi, \xi, \eta, g)$ onto a Riemannian manifold $\left(N, g^{\prime}\right)$ with slant angles $\theta_{1}, \theta_{2}$. Then $\left(\operatorname{ker} F_{*}\right)^{\perp}$ is integrable if and only if

$$
\begin{aligned}
g\left(\mathcal{A}_{Y} B X-\mathcal{A}_{X} B Y, \omega U\right)= & g\left(\mathcal{H} \nabla_{X} C Y-\mathcal{H} \nabla_{Y} C X, \omega U\right)+\eta(Y) g(Y, \omega U) \\
& -\eta(X) g(Y, \omega U)-g([X, Y], \omega \psi U)
\end{aligned}
$$

for $X, Y \in \Gamma\left(\left(\operatorname{ker} F_{*}\right)^{\perp}\right)$ and $U \in \Gamma\left(\operatorname{ker} F_{*}\right)$.

Proof. For $X, Y \in \Gamma\left(\left(\operatorname{ker} F_{*}\right)^{\perp}\right)$ and $U \in \Gamma\left(\operatorname{ker} F_{*}\right)$. Then since $M$ is a Sasakian manifold we get

$$
\begin{aligned}
g([X, Y], U)= & -g\left(\nabla_{X} Y, \phi \psi U\right)+g\left(\phi \nabla_{X} Y, \omega U\right) \\
& +g\left(\nabla_{Y} X, \phi \psi U\right)-g\left(\phi \nabla_{Y} X, \omega U\right) .
\end{aligned}
$$

From Theorem 3.5 we deduce that

$$
\begin{aligned}
\sin ^{2} \theta_{1} g([X, Y], U)= & \left(\cos ^{2} \theta_{2}-\cos ^{2} \theta_{1}\right) g([X, Y], Q U)-g\left(\nabla_{X} Y, \omega \psi U\right) \\
& +g\left(\nabla_{Y} X, \omega \psi U\right)+g\left(\nabla_{X} \phi Y, \omega U\right)+\eta(Y) g(X, \omega U) \\
& -g\left(\nabla_{Y} \phi X, \omega U\right)-\eta(X) g(Y, \omega U)
\end{aligned}
$$

Then from the equations (2.10) and (2.11), we have

$$
\begin{aligned}
\sin ^{2} \theta_{1} g([X, Y], U)= & \left(\cos ^{2} \theta_{2}-\cos ^{2} \theta_{1}\right) g([X, Y], Q U)-g([X, Y], \omega \psi U) \\
& +g\left(\mathcal{A}_{X} B Y, \omega U\right)+g\left(\mathcal{H} \nabla_{X} C Y, \omega U\right)-g\left(\mathcal{A}_{Y} B X, \omega U\right) \\
& -g\left(\mathcal{H} \nabla_{Y} C X, \omega U\right)-\eta(X) g(Y, \omega U)+\eta(Y) g(X, \omega U)
\end{aligned}
$$

which gives the desired equation.

Theorem 3.8. Let $F$ be a bi-slant $\xi^{\perp}$-Riemannian submersion from a Sasakian manifold $(M, \phi, \xi, \eta, g)$ onto a Riemannian manifold $\left(N, g^{\prime}\right)$ with slant angles $\theta_{1}, \theta_{2}$. Then the distribution $D_{1}$ defines a totally geodesic foliation if and only if

$$
g\left(\mathcal{T}_{U} \omega \psi V, W\right)=g\left(\mathcal{T}_{U} \omega V, \psi W\right)+g\left(\mathcal{H} \nabla_{U} \omega V, \omega W\right)
$$

and

$$
g\left(\mathcal{T}_{U} \omega V, B X\right)=g\left(\mathcal{H} \nabla_{U} \omega \psi V, X\right)-g\left(\mathcal{H} \nabla_{U} \omega V, C X\right)
$$

where $U, V \in \Gamma\left(D_{1}\right), W \in \Gamma\left(D_{2}\right)$ and $X \in \Gamma\left(\left(\operatorname{ker} F_{*}\right)^{\perp}\right)$.

Proof. From the equations (2.1), (2.2) and (3.2) for any $U, V \in \Gamma\left(D_{1}\right)$ and $W \in \Gamma\left(D_{2}\right)$ we can write

$$
\begin{aligned}
g\left(\nabla_{U} V, W\right) & =g\left(\phi \nabla_{U} V, \phi W\right) \\
& =-g\left(\phi \nabla_{U} \psi V, W\right)+g\left(\nabla_{U} \omega V, \phi W\right)
\end{aligned}
$$

Then Theorem 3.5 implies that

$$
\sin ^{2} \theta_{1} g\left(\nabla_{U} V, W\right)=-g\left(\nabla_{U} \omega \psi V, W\right)+g\left(\nabla_{U} \omega V, \phi W\right)
$$

Hence by using the equation (2.9) we have

$$
\begin{aligned}
\sin ^{2} \theta_{1} g\left(\nabla_{U} V, W\right) & =g\left(\mathcal{H}_{U} \omega V, \omega W\right)+g\left(\mathcal{T}_{U} \omega V, \psi W\right) \\
& -g\left(\mathcal{T}_{U} \omega \psi V, W\right) .
\end{aligned}
$$


which proves the first equation. On the other hand, for $X \in \Gamma\left(\left(\operatorname{ker} F_{*}\right)^{\perp}\right)$, we derive

$$
\begin{aligned}
g\left(\nabla_{U} V, X\right) & =g\left(\nabla_{U} \phi V, \phi X\right)+g(V, \phi U) \eta(X) \\
& =-g\left(\phi \nabla_{U} \psi V, X\right)+g\left(\nabla_{U} \omega V, \phi X\right)+g(V, \phi U) \eta(X) .
\end{aligned}
$$

Considering Theorem 3.5 we arrive at

$$
\sin ^{2} \theta_{1} g\left(\nabla_{U} V, X\right)=-g\left(\nabla_{U} \omega \psi V, X\right)+g\left(\nabla_{U} \omega V, \phi X\right) .
$$

From (2.9) we have

$$
\sin ^{2} \theta_{1} g\left(\nabla_{U} V, X\right)=-g\left(\mathcal{H} \nabla_{U} \omega \psi V, X\right)+g\left(\mathcal{H} \nabla_{U} \omega V, C X\right)+g\left(\mathcal{T}_{U} \omega V, B X\right)
$$

which gives the second equation.

Theorem 3.9. Let $F$ be a bi-slant $\xi^{\perp}$-Riemannian submersion from a Sasakian manifold $(M, \phi, \xi, \eta, g)$ onto a Riemannian manifold $\left(N, g^{\prime}\right)$ with slant angles $\theta_{1}, \theta_{2}$. Then the distribution $\mathrm{D}_{2}$ defines a totally geodesic foliation if and only if

$$
g\left(\mathcal{T}_{W} \omega \psi Z, U\right)=g\left(\mathcal{T}_{W} \omega Z, \psi U\right)+g\left(\mathcal{H} \nabla_{W} \omega Z, \omega U\right)
$$

and

$$
g\left(\mathcal{T}_{W} \omega Z, B X\right)=g\left(\mathcal{H} \nabla_{W} \omega \psi Z, X\right)-g\left(\mathcal{H} \nabla_{W} \omega Z, C X\right)
$$

where $U \in \Gamma\left(D_{1}\right), W, Z \in \Gamma\left(D_{2}\right)$ and $X \in \Gamma\left(\left(\operatorname{ker} F_{*}\right)^{\perp}\right)$.

Proof. By using similar method in Theorem 3.8 the proof of this theorem can be easily made.

Theorem 3.10. Let $F$ be a bi-slant $\xi^{\perp}$-Riemannian submersion from a Sasakian manifold $(M, \phi, \xi, \eta, g)$ onto a Riemannian manifold $\left(N, g^{\prime}\right)$ with slant angles $\theta_{1}, \theta_{2}$. Then the distribution $\left(\operatorname{ker} F_{*}\right)^{\perp}$ defines a totally geodesic foliation on $M$ if and only if

$$
\begin{aligned}
\left(\cos ^{2} \theta_{1}-\cos ^{2} \theta_{2}\right) g\left(\mathcal{A}_{X} Y, Q U\right) & =-g\left(\mathcal{H} \nabla_{X} Y, \omega \psi U\right)+g\left(\mathcal{A}_{X} B Y, \omega U\right) \\
& +g\left(\mathcal{H} \nabla_{X} C Y, \omega U\right)+\eta(Y)(X, \omega U)
\end{aligned}
$$

where $X, Y \in \Gamma\left(\operatorname{ker} F_{*}\right)^{\perp}$ and $U \in \Gamma\left(\operatorname{ker} F_{*}\right)$.

Proof. For $X, Y \in \Gamma\left(\operatorname{ker} F_{*}\right)^{\perp}$ and $U \in \Gamma\left(\operatorname{ker} F_{*}\right)$ we can write

$$
\begin{aligned}
g\left(\nabla_{X} Y, U\right) & =g\left(\phi \nabla_{X} Y, \phi U\right) \\
& =-g\left(\nabla_{X} Y, \phi \psi U\right)+g\left(\phi \nabla_{X} Y, \omega U\right)
\end{aligned}
$$

By using Theorem 3.5 we obtain

$$
\begin{aligned}
g\left(\nabla_{X} Y, U\right)= & \cos ^{2} \theta_{1} g\left(\nabla_{X} Y, P U\right)+\cos ^{2} \theta_{2} g\left(\nabla_{X} Y, Q U\right)-g\left(\nabla_{X} Y, \omega \psi U\right) \\
& +g\left(\phi \nabla_{X} Y, \omega U\right)
\end{aligned}
$$

From the equations $(2.10),(2.11)$ and $P U=U-Q U$ we have

$$
\begin{aligned}
\sin ^{2} \theta_{1} g\left(\nabla_{X} Y, U\right)= & \left(\cos ^{2} \theta_{2}-\cos ^{2} \theta_{1}\right) g\left(\mathcal{A}_{X} Y, Q U\right) \\
& -g\left(\mathcal{H} \nabla_{X} Y, \omega \psi U\right)+g\left(\mathcal{A}_{X} B Y, \omega U\right) \\
& +g\left(\mathcal{H} \nabla_{X} C Y, \omega U\right)+\eta(Y) g(X, \omega U)
\end{aligned}
$$

Thus we have the desired equation. 
Theorem 3.11. Let $F$ be a bi-slant $\xi^{\perp-R i e m a n n i a n ~ s u b m e r s i o n ~ f r o m ~ a ~ S a s a k i a n ~ m a n i-~}$ fold $(M, \phi, \xi, \eta, g)$ onto a Riemannian manifold $\left(N, g^{\prime}\right)$ with slant angles $\theta_{1}, \theta_{2}$. Then the distribution (ker $F_{*}$ ) defines a totally geodesic foliation on $M$ if and only if

$$
\begin{aligned}
\left(\cos ^{2} \theta_{1}-\cos ^{2} \theta_{2}\right) g\left(\mathcal{T}_{U} Q V, X\right)= & -g\left(\mathcal{H} \nabla_{U} \omega \psi V, X\right)+g\left(\mathcal{T}_{U} \omega V, B X\right) \\
& +g\left(\mathcal{H} \nabla_{U} \omega V, C X\right)
\end{aligned}
$$

where $X \in \Gamma\left(\operatorname{ker} F_{*}\right)^{\perp}$ and $U, V \in \Gamma\left(\operatorname{ker} F_{*}\right)$.

Proof. Suppose that $X \in \Gamma\left(\operatorname{ker} F_{*}\right)^{\perp}$ and $U, V \in \Gamma\left(\operatorname{ker} F_{*}\right)$. Then we get

$$
\begin{aligned}
g\left(\nabla_{U} V, X\right)= & g\left(\nabla_{U} P V, X\right)+g\left(\nabla_{U} Q V, X\right) \\
= & g\left(\phi \nabla_{U} P V, \phi X\right)+g(\phi U, P V) \eta(X)+g\left(\phi \nabla_{U} Q V, \phi X\right) \\
& +g(\phi U, Q V) \eta(X)
\end{aligned}
$$

Considering that $M$ is a Sasakian manifold we arrive

$$
\begin{aligned}
g\left(\nabla_{U} V, X\right)= & -g\left(\nabla_{U} \psi^{2} P V, X\right)-g\left(\nabla_{U} \psi^{2} Q V, X\right)-g\left(\nabla_{U} \omega \psi V, X\right) \\
& +g\left(\nabla_{U} \omega V, \phi X\right)
\end{aligned}
$$

From (2.8), (2.9) and Theorem 3.5 we obtain

$$
\begin{aligned}
\sin ^{2} \theta_{1} g\left(\nabla_{U} V, X\right)= & \left(\cos ^{2} \theta_{2}-\cos ^{2} \theta_{1}\right) g\left(\mathcal{T}_{U} Q V, X\right) \\
& -g\left(\mathcal{H} \nabla_{U} \omega \psi V, X\right)+g\left(\mathcal{H} \nabla_{U} \omega V, C X\right) \\
& +g\left(\mathcal{T}_{U} \omega V, B X\right)
\end{aligned}
$$

which shows our assertion.

Theorem 3.12. Let $F$ be a bi-slant $\xi^{\perp}$-Riemannian submersion from a Sasakian manifold $(M, \phi, \xi, \eta, g)$ onto a Riemannian manifold $\left(N, g^{\prime}\right)$ with slant angles $\theta_{1}, \theta_{2}$. Then $F$ is a totally geodesic map if and only if

$$
\begin{aligned}
\left(\cos ^{2} \theta_{2}-\cos ^{2} \theta_{1}\right) g\left(\mathcal{A}_{X} Q U, Y\right)= & g\left(C \mathcal{H} \nabla_{X} \omega U, Y\right)+g\left(\mathcal{H} \nabla_{X} \omega \psi U, Y\right) \\
& +g\left(\omega \mathcal{A}_{X} \omega U, Y\right)-g(U, \phi X) \eta(Y)
\end{aligned}
$$

and

$$
\begin{aligned}
\left(\cos ^{2} \theta_{1}-\cos ^{2} \theta_{2}\right) g\left(\mathcal{T}_{U} Q V, X\right)= & -g\left(\mathcal{H} \nabla_{U} \omega \psi V, X\right)+g\left(\mathcal{T}_{U} \omega V, B X\right) \\
& +g\left(\mathcal{H} \nabla_{U} \omega V, C X\right)
\end{aligned}
$$

where $X, Y \in \Gamma\left(\operatorname{ker} F_{*}\right)^{\perp}$ and $U, V \in\left(\operatorname{ker} F_{*}\right)$.

Proof. Firstly since $F$ is a Riemannian submersion for $X, Y \in \Gamma\left(\operatorname{ker} F_{*}\right)^{\perp}$ we have

$$
\left(\nabla F_{*}\right)(X, Y)=0 .
$$

Therefore for $X, Y \in \Gamma\left(\operatorname{ker} F_{*}\right)^{\perp}$ and $U, V \in\left(\operatorname{ker} F_{*}\right)$ it is enough to show that $\left(\nabla F_{*}\right)(U, V)=$ 0 and $\left(\nabla F_{*}\right)(X, U)=0$. So we can write

$$
g^{\prime}\left(\left(\nabla F_{*}\right)(X, U), F_{*} Y\right)=-g^{\prime}\left(F_{*}\left(\nabla_{X} U\right), F_{*} Y\right)=-g\left(\nabla_{X} U, Y\right) .
$$

Then we have

$$
g\left(\nabla_{X} U, Y\right)=-g\left(\nabla_{X} \phi \psi U, Y\right)+g\left(\nabla_{X} \omega U, \phi Y\right)+g(U, \phi X) \eta(Y)
$$

From the equations (2.10), (2.11) and Theorem 3.5 we obtain the first equation of Theorem 3.12

$$
\begin{aligned}
\sin ^{2} \theta_{1} g\left(\nabla_{X} U, Y\right)= & \left(\cos ^{2} \theta_{2}-\cos ^{2} \theta_{1}\right) g\left(\mathcal{A}_{X} Q U, Y\right)-g\left(C \mathcal{H} \nabla_{X} \omega U, Y\right) \\
& -g\left(\mathcal{H} \nabla_{X} \omega \psi U, Y\right)-g\left(\omega \mathcal{A}_{X} \omega U, Y\right)+g(U, \phi X) \eta(Y) .
\end{aligned}
$$


Also, for the second equation of Theorem 3.12 we have

$$
g^{\prime}\left(\left(\nabla F_{*}\right)(U, V), F_{*}\right)=-g\left(\nabla_{U} V, X\right) .
$$

Then using the equations (2.8) and (2.9), we arrive

$$
\begin{aligned}
g\left(\nabla_{U} V, X\right)= & \left(\cos ^{2} \theta_{2}-\cos ^{2} \theta_{1}\right) g\left(\mathcal{T}_{U} Q V, X\right)-g\left(\mathcal{H} \nabla_{U} \omega \psi V, X\right) \\
& +g\left(\mathcal{T}_{U} \omega V, B X\right)+g\left(\mathcal{H} \nabla_{U} \omega V, C X\right)
\end{aligned}
$$

which completes proof.

Theorem 3.13. Let $F$ be a bi-slant $\xi^{\perp}$-Riemannian submersion from a Sasakian manifold $(M, \phi, \xi, \eta, g)$ onto a Riemannian manifold $\left(N, g^{\prime}\right)$ with slant angles $\theta_{1}, \theta_{2}$. If $\omega$ is parallel then

i) $\mathcal{T}_{U} V=-\left(\sec ^{2} \theta_{1}\right) C \mathcal{T}_{U} \psi V$

ii) $\mathcal{T}_{W} Z=-\left(\sec ^{2} \theta_{2}\right) C \mathcal{T}_{W} \psi Z$

iii) The fibers of $F$ are $\left(D_{1}, D_{2}\right)$-mixed geodesic.

for $U, V \in \Gamma\left(D_{1}\right)$ and $W, Z \in \Gamma\left(D_{2}\right)$.

Proof. From the equation (3.7), if $\omega$ is parallel we have

$$
\mathcal{T}_{U} \psi V=C \mathcal{T}_{U} V
$$

for $U, V \in \Gamma\left(D_{1}\right)$. By writing $\psi V$ instead of $V$, we have $(i)$. The proof of $(i i)$ is calculated by applying the same way. Morever if $\omega$ is parallel, from the equation (3.7) we arrive

$$
C^{2} \mathcal{T}_{W} U=C\left(\mathcal{T}_{W} \psi U\right)=-\cos ^{2} \theta_{1} \mathcal{T}_{W} U
$$

and

$$
C^{2} \mathcal{T}_{U} W=C\left(\mathcal{T}_{U} \psi W\right)=-\cos ^{2} \theta_{2} \mathcal{T}_{U} W
$$

for $U \in \Gamma\left(D_{1}\right)$ and $W \in \Gamma\left(D_{2}\right)$. Then we get

$$
\cos ^{2} \theta_{1} \mathcal{T}_{W} U=\cos ^{2} \theta_{2} \mathcal{T}_{W} U
$$

Therefore the fibers are shown to be $\left(D_{1}, D_{2}\right)$-mixed geodesic.

\section{Decomposition theorems}

In this section we give decompositions theorems using the existence of bi-slant $\xi^{\perp}$ Riemannian submersion. We assume that $g$ is a Riemannian metric tensor on the manifold $M=M_{1} \times M_{2}$ and the canonical foliations $D_{M_{1}}$ and $D_{M_{2}}$ intersect vertically everywhere. Then $g$ is the metric tensor of a usual product of Riemannian manifold if and only if $D_{M_{1}}$ and $D_{M_{2}}$ are totally geodesic foliations.

Now we can write the following theorems by using Theorem 3.8-3.10,

Theorem 4.1. Let $F$ be a bi-slant $\xi^{\perp}$-Riemannian submersion from a Sasakian manifold $(M, \phi, \xi, \eta, g)$ onto a Riemannian manifold $\left(N, g^{\prime}\right)$ with slant angles $\theta_{1}, \theta_{2}$. Then $M$ is a locally product manifold of the form $M_{D_{1}} \times M_{D_{2}} \times M_{\left(\mathrm{ker} F_{*}\right)^{\perp}}$ if and only if

$$
\begin{aligned}
g\left(\mathcal{T}_{U} \omega \psi V, W\right) & =g\left(\mathcal{T}_{U} \omega V, \psi W\right)+g\left(\mathcal{H} \nabla_{U} \omega V, \omega W\right), \\
g\left(\mathcal{T}_{U} \omega V, B X\right) & =g\left(\mathcal{H} \nabla_{U} \omega \psi V, X\right)-g\left(\mathcal{H} \nabla_{U} \omega V, C X\right), \\
g\left(\mathcal{T}_{W} \omega \psi Z, U\right) & =g\left(\mathcal{T}_{W} \omega Z, \psi U\right)+g\left(\mathcal{H} \nabla_{W} \omega Z, \omega U\right), \\
g\left(\mathcal{T}_{W} \omega Z, B X\right) & =g\left(\mathcal{H} \nabla_{W} \omega \psi Z, X\right)-g\left(\mathcal{H} \nabla_{W} \omega Z, C X\right)
\end{aligned}
$$

and

$$
\begin{aligned}
\left(\cos ^{2} \theta_{1}-\cos ^{2} \theta_{2}\right) g\left(\mathcal{A}_{X} Y, Q U\right) & =-g\left(\mathcal{H} \nabla_{X} Y, \omega \psi U\right)+g\left(\mathcal{A}_{X} B Y, \omega U\right) \\
& +g\left(\mathcal{H} \nabla_{X} C Y, \omega U\right)+\eta(Y)(X, \omega U)
\end{aligned}
$$


for $U, V \in \Gamma\left(D_{1}\right), W, Z \in \Gamma\left(D_{2}\right)$ and $X, Y \in \Gamma\left(\left(\operatorname{ker} F_{*}\right)^{\perp}\right)$.

Theorem 4.2. Let $F$ be a bi-slant $\xi^{\perp-R i e m a n n i a n ~ s u b m e r s i o n ~ f r o m ~ a ~ S a s a k i a n ~ m a n i f o l d ~}$ $(M, \phi, \xi, \eta, g)$ onto a Riemannian manifold $\left(N, g^{\prime}\right)$ with slant angles $\theta_{1}, \theta_{2}$. Then $M$ is a locally product manifold of the form $M_{\mathrm{ker} F_{*}} \times M_{\left(\mathrm{ker} F_{*}\right)^{\perp}}$ if and only if

$$
\begin{aligned}
\left(\cos ^{2} \theta_{1}-\cos ^{2} \theta_{2}\right) g\left(\mathcal{T}_{U} Q V, X\right)= & -g\left(\mathcal{H} \nabla_{U} \omega \psi V, X\right)+g\left(\mathcal{T}_{U} \omega V, B X\right) \\
& +g\left(\mathcal{H} \nabla_{U} \omega V, C X\right)
\end{aligned}
$$

and

$$
\begin{aligned}
\left(\cos ^{2} \theta_{1}-\cos ^{2} \theta_{2}\right) g\left(\mathcal{A}_{X} Y, Q U\right)= & -g\left(\mathcal{H} \nabla_{X} Y, \omega \psi U\right)+g\left(\mathcal{A}_{X} B Y, \omega U\right) \\
& +g\left(\mathcal{H} \nabla_{X} C Y, \omega U\right)+\eta(Y)(X, \omega U)
\end{aligned}
$$

for $U, V \in \Gamma\left(D_{1}\right), W, Z \in \Gamma\left(D_{2}\right)$ and $X, Y \in \Gamma\left(\left(\operatorname{ker} F_{*}\right)^{\perp}\right)$.

Theorem 4.3. Let $F$ be a bi-slant $\xi^{\perp}$-Riemannian submersion from a Sasakian manifold $(M, \phi, \xi, \eta, g)$ onto a Riemannian manifold $\left(N, g^{\prime}\right)$ with slant angles $\theta_{1}, \theta_{2}$ such that $\left(\operatorname{ker} F_{*}\right)^{\perp}=\omega D_{1} \oplus \omega D_{2} \oplus\langle\xi\rangle$. Then $M$ is a locally product manifold of the form $M_{D_{1}} \times$ $M_{D_{2}} \times M_{\left(\operatorname{ker} F_{*}\right)^{\perp}}$ if and only if

$$
\begin{aligned}
g\left(\mathcal{T}_{U} \omega \psi V, W\right) & =g\left(\mathcal{T}_{U} \omega V, \psi W\right)+g\left(\mathcal{H} \nabla_{U} \omega V, \omega W\right), \\
g\left(\mathcal{T}_{U} \omega V, \phi X\right) & =g\left(\mathcal{H} \nabla_{U} \omega \psi V, X\right), \\
g\left(\mathcal{T}_{W} \omega \psi Z, U\right) & =g\left(\mathcal{T}_{W} \omega Z, \psi U\right)+g\left(\mathcal{H} \nabla_{W} \omega Z, \omega U\right), \\
g\left(\mathcal{T}_{W} \omega Z, \phi X\right) & =g\left(\mathcal{H} \nabla_{W} \omega \psi Z, X\right)
\end{aligned}
$$

and

$$
\begin{aligned}
\left(\cos ^{2} \theta_{1}-\cos ^{2} \theta_{2}\right) g\left(\mathcal{A}_{X} Y, Q U\right)= & -g\left(\mathcal{H} \nabla_{X} Y, \omega \psi U\right)+g\left(\mathcal{A}_{X} \phi Y, \omega U\right) \\
& +\eta(Y)(X, \omega U)
\end{aligned}
$$

for $U, V \in \Gamma\left(D_{1}\right), W, Z \in \Gamma\left(D_{2}\right)$ and $X, Y \in \Gamma\left(\left(\operatorname{ker} F_{*}\right)^{\perp}\right)$.

Theorem 4.4. Let $F$ be a bi-slant $\xi^{\perp}$-Riemannian submersion from a Sasakian manifold $(M, \phi, \xi, \eta, g)$ onto a Riemannian manifold $\left(N, g^{\prime}\right)$ with slant angles $\theta_{1}, \theta_{2}$ such that $\left(\operatorname{ker} F_{*}\right)^{\perp}=\omega D_{1} \oplus \omega D_{2} \oplus\langle\xi\rangle$. Then $M$ is a locally product manifold of the form $M_{\mathrm{ker} F_{*}} \times$ $M_{\left(\mathrm{ker} F_{*}\right)^{\perp}}$ if and only if

$$
\left(\cos ^{2} \theta_{1}-\cos ^{2} \theta_{2}\right) g\left(\mathcal{T}_{U} Q V, X\right)=-g\left(\mathcal{H} \nabla_{U} \omega \psi V, X\right)+g\left(\mathcal{T}_{U} \omega V, \phi X\right)
$$

and

$$
\begin{aligned}
\left(\cos ^{2} \theta_{1}-\cos ^{2} \theta_{2}\right) g\left(\mathcal{A}_{X} Y, Q U\right)= & -g\left(\mathcal{H} \nabla_{X} Y, \omega \psi U\right)+g\left(\mathcal{A}_{X} \phi Y, \omega U\right) \\
& +\eta(Y)(X, \omega U)
\end{aligned}
$$

for $U, V \in \Gamma\left(D_{1}\right), W, Z \in \Gamma\left(D_{2}\right)$ and $X, Y \in \Gamma\left(\left(\operatorname{ker} F_{*}\right)^{\perp}\right)$.

\section{References}

[1] M.A. Akyol and Y. Gündüzalp, On the geometry of conformal anti-invariant $\xi^{\perp}$ submersions, Int. J. Maps Math. 1 (1), 50-67, 2018.

[2] M.A. Akyol and R. Sarı, On semi-slant $\xi^{\perp}$-Riemannian submersions, Mediterr. J. Math. 14, 234 (20 pp), 2017.

[3] M.A. Akyol and B. Şahin, Conformal slant submersions, Hacet. J. Math. Stat. 48 (1), 28-44, 2019. 
[4] M.A. Akyol, R. Sarı and E. Aksoy, Semi-invariant $\xi^{\perp}$-Riemannian submersions from almost contact metric manifolds, Int. J. Geom. Methods Mod. Phys. 14 (5), 1750074, 2017.

[5] L.S. Alqahtani, M.S. Stankovic and S. Uddin, Warped product bi-slant submanifolds of cosymplectic manifolds, Filomat 31 (16), 5065-5071, 2017.

[6] S. Aykurt Sepet and M. Ergüt, Pointwise slant submersions from cosymplectic manifolds, Turkish J. Math. 40, 582-593, 2016.

[7] P. Baird and J.C. Wood, Harmonic morphisms between Riemannian manifolds, London Mathematical Society Monographs, Oxford University Press, Oxford, 2003.

[8] J.L. Cabrerizo, A. Carriazo, L.M. Fernandez and M. Fernandez, Slant submanifolds in Sasakian manifolds, Glasgow Math. J. 42, 125-138, 2000.

[9] A. Carriazo, Bi-slant immersions, In Proceeding of the ICRAMS, Kharagpur, India, 88-97, 2000.

[10] B.Y. Chen, Geometry of Slant Submanifolds, Katholieke Universiteit Leuven, Leuven, 1990.

[11] M. Falcitelly, S. Ianus and A.M. Pastore, Riemannian Submersions and Related Topics, World Scientific, River Edge, NJ, 2004.

[12] A. Gray, Pseudo-Riemannian almost product manifolds and submersions, Journal of Mathematics and Mechanics 16, 715-737, 1967.

[13] Y. Gündüzalp, Slant submersions from almost product Riemannian manifolds, Turkish J. Math. 37, 863-873, 2013.

[14] Y. Gündüzalp, Semi-slant submersions from almost product Riemannian manifolds, Demonstratio Math. 49 (3), 345-356, 2016.

[15] Y. Gündüzalp, Slant submersions in paracontact geometry, Hacet. J. Math. Stat. 49 (3), 822-834, 2020.

[16] İ. Küpeli Erken and C. Murathan, Slant Riemannian submersions from Sasakian manifolds, Arab. J. Math. Sci. 22, 250-264, 2016.

[17] J.W. Lee, Anti-invariant $\xi^{\perp}$-Riemannian submersions from almost contact manifolds. Hacet. J. Math. Stat. 42 (3), 231-241, 2013.

[18] J.W. Lee, Pointwise slant submersions, Bull. Korean Math. Soc. 51 (4), 1115-1126, 2014.

[19] B. O'Neill, The fundamental equations of a submersion, Michigan Math. J. 13, 458469, 1966.

[20] F. Özdemir, C. Sayar and H.M. Taştan, Semi-invariant submersions whose total manifolds are locally product Riemannian, Quaest. Math. 40 (7), 909-926, 2017.

[21] K.S. Park and R. Prasad, Semi-slant submersions, Bull. Korean Math. Soc. 50 (3), 951-962, 2013.

[22] R. Prasad, S.S. Shukla and S. Kumar, On Quasi-bi-slant submersions, Mediterr. J. Math. 16, 155 (18 pp), 2019.

[23] C. Sayar, M.A. Akyol and R. Prasad, Bi-slant submersions in complex geometry, Int. J. Geom. Methods Mod. Phys. 17 (4), 2050055, 2020.

[24] C. Sayar, H.M. Taştan, F. Özdemir and M.M. Tripathi, Generic submersions from Kaehler Manifolds, Bull. Malays. Math. Sci. Soc. 43, 809-831, 2020.

[25] B. Şahin, Anti-invariant Riemannian submersions from almost Hermitian manifolds, Cent. Eur. J. Math. 8 (3), 437-447, 2010.

[26] B. Şahin, Semi-invariant Riemannian submersions from almost Hermitian manifolds, Canad. Math. Bull. 56 (1), 173-183, 2011.

[27] B. Şahin, Slant submersions from almost Hermitian manifolds, Bull. Math. Soc. Sci. Math. Roumanie 54 (102), 93-105, 2011.

[28] B. Şahin, Riemannian submersions, Riemannian maps in Hermitian geometry and their applications, Amsterdam, Netherlands: Elsevier Science Publishing Co., Inc., 2017. 
[29] H.M. Taştan, On Lagrangian submersions, Hacet. J. Math. Stat. 43 (6), 993-1000, 2014.

[30] H.M. Taştan, B. Şahin and Ş. Yanan, Hemi-slant submersions, Mediterr. J. Math. 13, 2171-2184, 2016.

[31] S. Uddin, B.Y. Chen and F.R. Al-Solamy, Warped Product Bi-slant Immersions in Kaehler Manifolds, Mediterr. J. Math. 14, 95 (11 pages), 2017.

[32] B. Watson, Almost Hermitian submersions, J. Differential Geom. 11 (1), 147-165, 1976. 